\title{
Norm-attaining polynomials and differentiability
}

\author{
by \\ JuAN FERRERA (Madrid)
}

\begin{abstract}
We give a polynomial version of Shmul'yan's Test, characterizing the polynomials that strongly attain their norm as those at which the norm is Fréchet differentiable. We also characterize the Gateaux differentiability of the norm. Finally we study those properties for some classical Banach spaces.
\end{abstract}

1. Introduction. Throughout this paper $E$ will be a Banach space over $\mathbb{R}, E^{*}$ will denote the dual space, and $E^{* *}$ the bidual. The unit sphere and the closed unit ball of $E$ will be denoted by $S_{E}$ and $B_{E}$ respectively. A continuous function $P: E \rightarrow \mathbb{R}$ is a $k$-homogeneous polynomial provided that there exists a $k$-linear form $\widehat{P}$ on $E$ such that $P(x)=\widehat{P}(x, \ldots, x)$; $\mathcal{P}\left({ }^{k} E\right)$ will denote the space of all $k$-homogeneous continuous polynomials on $E$. We define the norm on $\mathcal{P}\left({ }^{k} E\right)$ in the usual way:

$$
\|P\|=\sup \{|P(x)|:\|x\| \leq 1\} \text {. }
$$

This norm satisfies

$$
\|P\| \leq\|\widehat{P}\| \leq \frac{k^{k}}{k !}\|P\|
$$

(see [12], [15], [17] or [18] for a general reference on infinite-dimensional polynomials).

The vector subspace generated by all the polynomials $f^{k}$ where $f \in E^{*}$ will be denoted by $\mathcal{P}_{\mathrm{f}}\left({ }^{k} E\right)$; we will refer to the polynomials belonging to $\mathcal{P}_{\mathrm{f}}\left({ }^{k} E\right)$ as finite type polynomials. The closure of $\mathcal{P}_{\mathrm{f}}\left({ }^{k} E\right)$ will be denoted by $\mathcal{P}_{\mathrm{c}}\left({ }^{k} E\right)$.

We denote by $\mathcal{P}_{\text {wsc }}\left({ }^{k} E\right)$, respectively $\mathcal{P}_{\text {wb }}\left({ }^{k} E\right)$, the space of all $P \in \mathcal{P}\left({ }^{k} E\right)$ which are weakly sequentially continuous, respectively weakly continuous when restricted to bounded sets. We have

$$
\mathcal{P}_{\mathrm{c}}\left({ }^{k} E\right) \subset \mathcal{P}_{\mathrm{wb}}\left({ }^{k} E\right) \subset \mathcal{P}_{\mathrm{wsc}}\left({ }^{k} E\right) .
$$

2000 Mathematics Subject Classification: Primary 46G25; Secondary 46B20.

Research partially supported by DGICYT project PB-0044 (Spain) and Fundación DEL AMO (UCM, Spain). 
Moreover, $\mathcal{P}_{\mathrm{wb}}\left({ }^{k} E\right)=\mathcal{P}_{\mathrm{wsc}}\left({ }^{k} E\right)$ if and only if $E$ does not contain $l_{1}$ (see [14]), while $\mathcal{P}_{\mathrm{c}}\left({ }^{k} E\right)=\mathcal{P}_{\mathrm{wb}}\left({ }^{k} E\right)$ provided that $E^{*}$ has the approximation property.

The Aron-Berner extension (see [4], [16]) provides us with a linear map

$$
L: \mathcal{P}\left({ }^{k} E\right) \rightarrow \mathcal{P}\left({ }^{k} E^{* *}\right), \quad L(P)=\widetilde{P},
$$

such that $\widetilde{P}=P$ for every $P \in \mathcal{P}_{\mathrm{f}}\left({ }^{k} E\right)$, in the following sense:

$$
L\left(f^{k}\right)\left(x^{* *}\right)=x^{* *}(f)^{k} .
$$

In particular we have:

Lemma 1.1. If $P \in \mathcal{P}_{\mathrm{f}}\left({ }^{k} E\right)$, then $\widetilde{P}$ is $w^{*}$-continuous.

The Aron-Berner extension is continuous, in fact $\|P\|=\|\widetilde{P}\|$ (see [9]). From this it is easy to deduce the following

Lemma 1.2. If $P \in \mathcal{P}_{\mathrm{c}}\left({ }^{k} E\right)$, then $\widetilde{P}$ is $w^{*}$-continuous on bounded sets.

Proof. We have $P=\lim _{n} P_{n}$ where $P_{n} \in \mathcal{P}_{\mathrm{f}}\left({ }^{k} E\right)$. By continuity of AronBerner extension, we have $\widetilde{P}=\lim _{n} \widetilde{P}_{n}$.

We will say that a polynomial $P \in \mathcal{P}\left({ }^{k} E\right)$ attains its norm if there exists an $x_{0} \in S_{E}$ such that $\|P\|=\left|P\left(x_{0}\right)\right|$. The Bishop-Phelps Theorem asserts that the set of all linear functionals which attain their norm is a dense subset of the dual space. As shown in [1] this is not a general fact for Banach spaces if $k>1$. For positive results see [8]. A related, but not equivalent, problem is that of norm-attaining multilinear forms; see [5]. Sometimes polynomials "attain their norm" in the bidual.

Proposition 1.3. If $P \in \mathcal{P}_{\mathrm{c}}\left({ }^{k} E\right)$, then $\widetilde{P}$ attains its norm at a point $x_{0}^{* *} \in S_{E^{* *}}$.

Proof. This follows from Lemma 1.2 and $w^{*}$-compactness of $B_{E^{* *}}$.

In particular, if $E$ does not contain a copy of $l_{1}$ and $E^{*}$ has the approximation property, then the Aron-Berner extension of every weakly sequentially continuous polynomial attains its norm. This is not true in general, even for weakly sequentially continuous polynomials, as the following example proves for the Banach space $l_{1}$.

Example. We define $P \in \mathcal{P}\left({ }^{2} l_{1}\right)$ by

$$
P(x)=\sum_{n=1}^{\infty}\left(1-2^{-n}\right) e_{n}^{*}(x)^{2} .
$$

Since $l_{1}$ is a Schur space, $P$ is weakly sequentially continuous. Moreover, $\|P\|=1$ but $|P(x)|<1$ for every $x \in S_{l_{1}}$, hence $P$ does not attain its norm. 
The Aron-Berner extension is defined by

$$
\widetilde{P}\left(x^{* *}\right)=\sum_{n=1}^{\infty}\left(1-2^{-n}\right) e_{n}^{*}\left(x^{* *}\right)^{2}
$$

since if $A$ is the symmetric $k$-linear form associated to $P, x^{* *}$ and $y^{* *}$ two points in $B_{E^{* *}}$, and $\left\{x_{\alpha}\right\}$ and $\left\{y_{\beta}\right\}$ two nets in $B_{E}$ converging to $x^{* *}$ and $y^{* *}$ respectively, then

$$
\begin{aligned}
\widetilde{A}\left(x^{* *}, y^{* *}\right) & =\lim _{\alpha} \lim _{\beta} A\left(x_{\alpha}, y_{\beta}\right)=\lim _{\alpha} \lim _{\beta} \sum_{n=1}^{\infty}\left(1-2^{-n}\right) e_{n}^{*}\left(x_{\alpha}\right) e_{n}^{*}\left(y_{\beta}\right) \\
& =\sum_{n=1}^{\infty}\left(1-2^{-n}\right) e_{n}^{*}\left(x^{* *}\right) e_{n}^{*}\left(y^{* *}\right)
\end{aligned}
$$

by the Lebesgue Dominated Convergence Theorem.

We may represent every $x^{* *} \in l_{1}^{* *}$ as $x+\widetilde{x}$, where $x \in l_{1}$ and $\widetilde{x}$ vanishes on $c_{0}$. Hence $e_{n}^{*}\left(x^{* *}\right)=e_{n}^{*}(x)$, and $\left|\widetilde{P}\left(x^{* *}\right)\right|=|P(x)|<\|P\|=\|\widetilde{P}\|$ provided that $\left\|x^{* *}\right\| \leq 1$, since $\|x\|_{1} \leq\left\|x^{* *}\right\|$. Therefore $\widetilde{P}$ does not attain its norm.

The following easy corollary may be seen as a polynomial version of the James Theorem.

Corollary 1.4. A Banach space $E$ is reflexive if and only if every $P \in$ $\mathcal{P}_{\mathrm{c}}\left({ }^{k} E\right)$ attains its norm.

In this paper we introduce a stronger way of attaining the norm, which will allow us to give characterizations in terms of the differentiability of the $\mathcal{P}\left({ }^{k} E\right)$ norm.

Definition 1.5. We will say that a polynomial $P \in \mathcal{P}\left({ }^{k} E\right)$ strongly attains its norm whenever there exists an $x_{0} \in S_{E}$ such that every sequence $\left\{x_{n}\right\} \subset S_{E}$ satisfying $\|P\|=\lim _{n}\left|P\left(x_{n}\right)\right|$ has a subsequence that converges either to $x_{0}$ or to $-x_{0}$.

It is clear that if a polynomial $P$ attains its norm strongly, then so does $\lambda P$ for every nonzero $\lambda \in \mathbb{R}$. We will refer to the sequences such that $\lim _{n}\left|P\left(x_{n}\right)\right|=\|P\|$ as approaching sequences; it is easy to see that if $P$ strongly attains its norm then each approaching sequence either converges or may be split in two subsequences $\left\{x_{n}^{+}\right\}$and $\left\{x_{n}^{-}\right\}$converging to $x_{0}$ and $-x_{0}$ respectively.

Observe that if $k$ is even, then either every approaching sequence $\left\{x_{n}\right\}$ satisfies $\lim _{n} P\left(x_{n}\right)=\|P\|$, or every approaching sequence $\left\{x_{n}\right\}$ satisfies $\lim _{n} P\left(x_{n}\right)=-\|P\|$. On the other hand, if $k$ is odd and $\lim _{n} P\left(x_{n}\right)= \pm\|P\|$, then $\left\{x_{n}\right\}$ is convergent (either to $x_{0}$ or to $-x_{0}$ ). 
2. Fréchet differentiability. We start with a definition that will be useful in the proof of the main theorem of this section.

Definition 2.1. We will say that a polynomial $P \in \mathcal{P}\left({ }^{k} E\right)$ strongly approaches its norm whenever for any sequences $\left\{x_{n}\right\} \subset S_{E}$ and $\left\{y_{n}\right\} \subset S_{E}$ satisfying

$$
\lim _{n} P\left(x_{n}\right)=s_{x}\|P\|, \quad \lim _{n} P\left(y_{n}\right)=s_{y}\|P\|, \quad s_{x}, s_{y} \in\{+1,-1\},
$$

we have $\lim _{n}\left(s_{x} Q\left(x_{n}\right)-s_{y} Q\left(y_{n}\right)\right)=0$ uniformly in $Q \in S_{\mathcal{P}\left({ }^{k} E\right)}$.

As above, if a polynomial $P$ strongly approaches its norm, the polynomials $\lambda P$ have the same property for every nonzero $\lambda \in \mathbb{R}$.

Observe that if $k$ is even, then necessarily $s_{x} s_{y}=1$ in the definition above. Otherwise $\lim _{n}\left(Q\left(x_{n}\right)+Q\left(y_{n}\right)\right)=0$ uniformly in $Q \in S_{\mathcal{P}\left({ }^{k} E\right)}$. In particular $\lim _{n}\left(x^{*}\left(x_{n}\right)^{k}+x^{*}\left(y_{n}\right)^{k}\right)=0$ uniformly in $x^{*} \in S_{E^{*}}$ and, $k$ being even, $\lim _{n} x^{*}\left(x_{n}\right)^{k}=0$ uniformly in $x^{*} \in S_{E^{*}}$, and hence $\lim _{n}\left\|x_{n}\right\|=0$, which is not possible.

On the other hand, if $k$ is odd the definition reads: Given two sequences $\left\{x_{n}\right\}$ and $\left\{y_{n}\right\}$ in the unit sphere such that $\|P\|=\lim _{n} P\left(x_{n}\right)=\lim _{n} P\left(y_{n}\right)$, we necessarily have $\lim _{n}\left(Q\left(x_{n}\right)-Q\left(y_{n}\right)\right)=0$ uniformly in $Q \in S_{\mathcal{P}\left({ }^{k} E\right)}$.

As a matter of fact Definitions 1.5 and 2.1 are equivalent, but in order to prove this we need the following lemma.

Lemma 2.2. Let $E$ be a Banach space. Then for every $x, y \in E$ there exists a $g \in S_{E^{*}}$ such that $\left|g(x)^{2}-g(y)^{2}\right| \geq \frac{1}{4}\|x+y\| \cdot\|x-y\|$.

Proof. It is equivalent to prove that for every $x, y \in S_{E}$ there exists a $g \in S_{E^{*}}$ such that $|g(x) g(y)| \geq 1 / 4$. This is an easy consequence of the following geometric result:

Consider two linearly independent points $A, B$ in the real plane, and the parallelogram $\operatorname{co}\{A,-A, B,-B\}$. For every pair of points $C \in \overline{A B}, D \in$ $\overline{A(-B)}$, at least two vertices of $\frac{1}{2} \operatorname{co}\{A, B,-A,-B\}$ are contained in the parallelogram $\operatorname{co}\{C, D,-C,-D\}$.

In order to prove the lemma we start with two points $x, y \in S_{E}$. Let $f_{y}, f_{x} \in S_{E^{*}}$ be such that $f_{x}(x)=1$ and $f_{y}(y)=1$. We are going to work in the two-dimensional space $X=E^{*} \cap\left[\left\{f_{x}, f_{y}\right\}\right] ; B^{*}$ will denote its unit ball. Let $A, B,-A$ and $-B$ denote the four vertices of the parallelogram generated by the lines $x=1, x=-1, y=1, y=-1$. It is clear that $B^{*} \subset \operatorname{co}\{A, B,-A,-B\}$. Denote $f_{x}$ by $C$, and $f_{y}$ by $D$. The parallelogram $\operatorname{co}\{C, D,-C,-D\}$ is a subset of $B^{*}$ by convexity. The vertex $g$ of $\frac{1}{2} \operatorname{co}\{A, B,-A,-B\}$ contained in $\operatorname{co}\{C, D,-C,-D\} \subset B^{*}$ satisfies $g(x)= \pm 1 / 2$ and $g(y)= \pm 1 / 2$.

The geometric claim is easily checked if the parallelogram is a square, but this is enough because the claim is linearly invariant. 
The constant $1 / 4$ is sharp as the following easy example shows: $E=l_{1}^{2}$, $x=(1,0), y=(0,1)$. For Hilbert spaces the constant is $1 / 2$, as a matter of fact this characterizes Hilbert spaces (see [6]). On the other hand, it is clear that if $E$ is a dual space, then we may choose the linear functional $g$ in the predual, although we will have to change the constant $1 / 4$ to $1 / 4-\varepsilon$. (Check the constant for $E=l_{1}, x=\sum_{n=1}^{\infty} 2^{-n} e_{2 n}$ and $y=\sum_{n=1}^{\infty} 2^{-n} e_{2 n-1}$.)

We are now ready to prove the equivalence of both definitions.

Proposition 2.3. $P \in \mathcal{P}\left({ }^{k} E\right)$ strongly attains its norm if and only if it strongly approaches its norm.

Proof. It is easy to see that if $P$ attains its norm strongly, then it approaches it strongly. Indeed, suppose first that $k$ is odd. If $\left\{x_{n}\right\},\left\{y_{n}\right\}$ are sequences in the unit sphere such that $\lim _{n} P\left(x_{n}\right)=\|P\|$ and $\lim _{n} P\left(y_{n}\right)=$ $\|P\|$, then both converge to the same $x_{0}$. Now, if $\|Q\|=1$, by the Mean Value Theorem, we have

$$
\left|Q\left(x_{n}\right)-Q\left(y_{n}\right)\right| \leq \frac{k^{k+1}}{k !}\left\|x_{n}-y_{n}\right\|
$$

since if $\widehat{Q}$ is the $k$-linear symmetric form associated to $Q$, then $\|\widehat{Q}\| \leq k^{k} / k$ !. The last term goes to 0 , independently of $Q$, and therefore the convergence is uniform in $Q \in S_{\mathcal{P}\left({ }^{k} E\right)}$.

If $k$ is even, from the fact that $P$ attains its norm strongly it follows that $s_{x} s_{y}=1$. Define $\alpha_{n}, \beta_{n} \in\{-1,1\}$ to be such that $\lim _{n} \alpha_{n} x_{n}=\lim _{n} \beta_{n} y_{n}$ $=x_{0}$. We have

$$
\begin{aligned}
\left|s_{x} Q\left(x_{n}\right)-s_{y} Q\left(y_{n}\right)\right| & =\left|Q\left(x_{n}\right)-Q\left(y_{n}\right)\right|=\left|Q\left(\alpha_{n} x_{n}\right)-Q\left(\beta_{n} y_{n}\right)\right| \\
& \leq \frac{k^{k+1}}{k !}\left\|\alpha_{n} x_{n}-\beta_{n} y_{n}\right\|,
\end{aligned}
$$

which goes to 0 uniformly in $Q \in S_{\mathcal{P}\left({ }^{k} E\right)}$.

Conversely, assume first that $k$ is even. Take an approaching sequence $\left\{x_{n}\right\}$. Considering $-P$ instead of $P$ if necessary, we may assume that $\lim _{n} P\left(x_{n}\right)=\|P\|$. We are going to prove that $\left\{x_{n}\right\}$ has a Cauchy subsequence. This is enough because if two subsequences $\left\{y_{n}\right\},\left\{z_{n}\right\}$ converge to $y_{0}$ and $z_{0}$ respectively, then $P\left(y_{0}\right)=P\left(z_{0}\right)=\|P\|$ and consequently $Q\left(y_{0}\right)=Q\left(z_{0}\right)$ for every $Q$ such that $\|Q\|=1$, which implies that either $y_{0}=z_{0}$ or $y_{0}=-z_{0}$.

Let $D$ denote the range of the sequence $\left\{x_{n}\right\}$; we may assume that it is infinite (otherwise there is a trivial Cauchy subsequence). Define $A_{n}=$ $\bar{B}\left(x_{n}, 1 / 2\right) \cup \bar{B}\left(-x_{n}, 1 / 2\right)$. If $D \cap A_{n}$ is finite for every $n$, we may define a subsequence in the following way: 


$$
\begin{gathered}
x_{n_{1}}=x_{1}, \quad A_{n_{1}}=A_{1}, \\
x_{n_{2}} \notin A_{n_{1}}, \quad n_{2}>n_{1}, \\
\ldots \ldots \ldots \\
x_{n_{r}} \notin A_{n_{1}} \cup \ldots \cup A_{n_{r-1}}, \quad n_{r}>n_{r-1} .
\end{gathered}
$$

This sequence has the property that if $j \neq i$, then $\left\|x_{n_{j}}-x_{n_{i}}\right\|$ and $\left\|x_{n_{j}}+x_{n_{i}}\right\|$ are greater than $1 / 2$. If we now define $x_{r}=x_{n_{2 r-1}}$ and $y_{r}=x_{n_{2 r}}$, we see that $\min \left\{\left\|x_{r}-y_{r}\right\|,\left\|x_{r}+y_{r}\right\|\right\}>1 / 2$ for every $r$, and by the lemma above, for every $r$ there exists a $g_{r} \in S_{E^{*}}$ such that $\left|g_{r}\left(x_{r}\right)^{2}-g_{r}\left(y_{r}\right)^{2}\right| \geq 1 / 8$, hence $\left|g_{r}\left(x_{r}\right)^{k}-g_{r}\left(y_{r}\right)^{k}\right| \geq(1 / 8)^{k / 2}$. Therefore $\left\{Q\left(x_{r}\right)-Q\left(y_{r}\right)\right\}$ does not converge to 0 uniformly in $S_{\mathcal{P}\left({ }^{k} E\right)}$, which gives us a contradiction, because the sequences fulfil the conditions of Definition 2.1.

Therefore there exists an $n_{0}$ such that either $D \cap \bar{B}\left(x_{n_{0}}, 1 / 2\right)$ or $D \cap$ $\bar{B}\left(-x_{n_{0}}, 1 / 2\right)$ is infinite. In either case there exists a subsequence of $\left\{x_{n}\right\}$, still denoted by $\left\{x_{n}\right\}$, such that $\left\|x_{n}-x_{m}\right\| \leq 1$ for all $n, m$, and $\left\|x_{n}+x_{m}\right\|$ $\geq 1$ since $x_{n}, x_{m} \in S_{E}$. If it is not a Cauchy sequence, there exists a positive $\varepsilon$ such that for every $j$, there exist $n_{j}, m_{j}$ with $n_{j}>n_{j-1}$ and $m_{j}>m_{j-1}$ such that $\left\|x_{m_{j}}-x_{n_{j}}\right\|>\varepsilon$. Set $y_{j}=x_{n_{j}}$ and $z_{j}=x_{m_{j}}$. These sequences have the property that for every $j,\left\|y_{j}+z_{j}\right\| \geq 1$ and $\left\|y_{j}-z_{j}\right\| \geq \varepsilon$, hence, by the lemma again, we have a contradiction.

For $k$ odd, it is again enough to prove that every approaching sequence $\left\{x_{n}\right\}$ has a Cauchy subsequence. Passing to a subsequence if necessary, we may assume that $\lim _{n} P\left(x_{n}\right)=\|P\|$. If $\left\{x_{n}\right\}$ is not a Cauchy sequence, we may choose two subsequences $\left\{y_{j}\right\}$ and $\left\{z_{j}\right\}$, and a positive $\varepsilon$, such that $\left\|y_{j}-z_{j}\right\|>\varepsilon$ for every $j$. Therefore for every $j$ there exists a $Q_{j} \in S_{\mathcal{P}\left({ }^{k} E\right)}$, $Q_{j}=\left(f_{j}\right)^{k}$ for a suitable $f_{j} \in S_{E^{*}}$, such that $\left|Q_{j}\left(y_{j}\right)-Q_{j}\left(z_{j}\right)\right|>2(\varepsilon / 2)^{k}$, and consequently $P$ does not approach its norm strongly.

The following theorem is a sort of Shmul'yan's Test, and gives us a characterization of the polynomials that strongly attain their norm by means of the differentiability of the norm.

TheOREM 2.4. $P \in \mathcal{P}\left({ }^{k} E\right)$ strongly attains its norm if and only if \|\| : $\mathcal{P}\left({ }^{k} E\right) \rightarrow \mathbb{R}$ is Fréchet differentiable at $P$.

Proof. Suppose first that $P$ strongly attains its norm, and assume, without loss of generality, that $\|P\|=1$. If \|\|$: \mathcal{P}\left({ }^{k} E\right) \rightarrow \mathbb{R}$ is not Fréchet differentiable at $P$, then by the standard characterization of Fréchet differentiability for convex functions (see [10]), there exist an $\varepsilon>0$ and a sequence $\left\{Q_{n}\right\} \subset \mathcal{P}\left({ }^{k} E\right)$ converging to $0\left(Q_{n} \neq 0\right)$ such that

$$
\left\|P+Q_{n}\right\|+\left\|P-Q_{n}\right\| \geq 2+\varepsilon\left\|Q_{n}\right\| .
$$

Let $x_{n} \in S_{E}$ be such that $\left|\left(P+Q_{n}\right)\left(x_{n}\right)\right| \geq\left\|P+Q_{n}\right\|-\frac{1}{n}\left\|Q_{n}\right\|$. It follows 
that

$$
\begin{aligned}
1 & \geq\left|P\left(x_{n}\right)\right|=\left|\left(P+Q_{n}\right)\left(x_{n}\right)-Q_{n}\left(x_{n}\right)\right| \\
& \geq\left|\left(P+Q_{n}\right)\left(x_{n}\right)\right|-\left|Q_{n}\left(x_{n}\right)\right| \\
& \geq|| P+Q_{n}\left\|-\frac{1}{n}\right\| Q_{n} \|-\left|Q_{n}\left(x_{n}\right)\right|,
\end{aligned}
$$

hence $\lim _{n}\left|P\left(x_{n}\right)\right|=1$. Similarly, if $y_{n} \in S_{E}$ satisfies $\left|\left(P-Q_{n}\right)\left(y_{n}\right)\right| \geq$ $\left\|P-Q_{n}\right\|-\frac{1}{n}\left\|Q_{n}\right\|$, then $\lim _{n}\left|P\left(y_{n}\right)\right|=1$. Passing to a subsequence if necessary, we may assume that $\lim _{n} P\left(x_{n}\right)=s_{x}$ and $\lim _{n} P\left(y_{n}\right)=s_{y}$, with $s_{x}, s_{y} \in\{+1,-1\}$. Hence

$$
\begin{aligned}
& s_{x}\left(P+Q_{n}\right)\left(x_{n}\right) \geq\left\|P+Q_{n}\right\|-\frac{1}{n}\left\|Q_{n}\right\|, \\
& s_{y}\left(P-Q_{n}\right)\left(y_{n}\right) \geq\left\|P-Q_{n}\right\|-\frac{1}{n}\left\|Q_{n}\right\|,
\end{aligned}
$$

since $\operatorname{sign}\left[\left(P+Q_{n}\right)\left(x_{n}\right)\right]=s_{x}$ and $\operatorname{sign}\left[\left(P-Q_{n}\right)\left(y_{n}\right)\right]=s_{y}$ if $n$ is large enough. Therefore

$$
\begin{aligned}
s_{x}\left(P+Q_{n}\right) & \left(x_{n}\right)+s_{y}\left(P-Q_{n}\right)\left(y_{n}\right) \\
& \geq\left\|P+Q_{n}\right\|+\left\|P-Q_{n}\right\|-\frac{2}{n}\left\|Q_{n}\right\| \geq 2+\left(\varepsilon-\frac{2}{n}\right)\left\|Q_{n}\right\|,
\end{aligned}
$$

and consequently

$$
s_{x} Q_{n}\left(x_{n}\right)-s_{y} Q_{n}\left(y_{n}\right) \geq\left(\varepsilon-\frac{2}{n}\right)\left\|Q_{n}\right\|,
$$

which is impossible because $\lim _{n}\left(s_{x} Q\left(x_{n}\right)-s_{y} Q\left(y_{n}\right)\right)=0$ uniformly in $Q \in S_{\mathcal{P}\left({ }^{k} E\right)}$.

Conversely, once again we suppose, without loss of generality, that $\|P\|$ $=1$. Take sequences $\left\{x_{n}\right\},\left\{y_{n}\right\}$ in the unit sphere of $E$ such that $\lim _{n} P\left(x_{n}\right)$ $=s_{x}$ and $\lim _{n} P\left(y_{n}\right)=s_{y}$ where $s_{x}, s_{y} \in\{-1,+1\}$.

By Fréchet differentiability, for every $\varepsilon>0$ there exists a $\delta>0$ such that if $\|Q\|<\delta$ then

$$
\|P+Q\|+\|P-Q\| \leq 2+\varepsilon\|Q\|
$$

and consequently

$$
\left|(P+Q)\left(x_{n}\right)\right|+\left|(P-Q)\left(y_{n}\right)\right| \leq 2+\varepsilon\|Q\| .
$$

If $\delta$ is small enough, then $\left|(P+Q)\left(x_{n}\right)\right|=s_{x}(P+Q)\left(x_{n}\right)$ and $\left|(P-Q)\left(y_{n}\right)\right|=$ $s_{y}(P-Q)\left(y_{n}\right)$, hence

$$
\begin{aligned}
s_{x} P\left(x_{n}\right)+s_{y} P\left(y_{n}\right)+ & \left(s_{x} Q\left(x_{n}\right)-s_{y} Q\left(y_{n}\right)\right) \\
& =s_{x}(P+Q)\left(x_{n}\right)+s_{y}(P-Q)\left(y_{n}\right) \leq 2+\varepsilon\|Q\| .
\end{aligned}
$$


From $\lim _{n}\left(s_{x} P\left(x_{n}\right)+s_{y} P\left(y_{n}\right)\right)=2$, it follows that there exists $n_{0}$ such that if $n>n_{0}$ then $2-\left(s_{x} P\left(x_{n}\right)+s_{y} P\left(y_{n}\right)\right)<\varepsilon \delta$, and consequently

$$
s_{x} Q\left(x_{n}\right)-s_{y} Q\left(y_{n}\right) \leq 2 \varepsilon \delta \quad \forall n>n_{0} \forall Q \text { with }\|Q\|<\delta,
$$

or equivalently $\lim _{n}\left(s_{x} Q\left(x_{n}\right)-s_{y} Q\left(y_{n}\right)\right)=0$ uniformly on $S_{\mathcal{P}\left({ }^{k} E\right)}$. So $P$ strongly approaches its norm and, by the proposition above, it attains it strongly.

COROLlary 2.5. The set of all polynomials which strongly attain their norm is $a G_{\delta}$ subset of $\mathcal{P}\left({ }^{k} E\right)$.

Proof. It is enough to observe that the set of points at which a convex function is Fréchet differentiable is always a $G_{\delta}$ subset. See e.g. [10].

Recall that a subset $D$ of a Banach space $E$ is dentable if for every positive $\varepsilon$ there exists an $x_{\varepsilon} \in D$ such that $x_{\varepsilon} \notin \overline{\mathrm{co}}\left(D-B\left(x_{\varepsilon}, \varepsilon\right)\right.$ ), and that a Banach space has the Radon-Nikodym Property (RNP) whenever every nonempty bounded set is dentable. In [8], it is proved that if a Banach space $E$ has RNP, then the set of norm-attaining polynomials is dense. Here, modifying slightly their proof, and using the corollary above we have:

THEOREM 2.6. If the Banach space $E$ has the RNP, then the set of all polynomials which attain their norm strongly is a $G_{\delta}$ dense subset of $\mathcal{P}\left({ }^{k} E\right)$.

Proof. We only have to prove that it is dense. Proceeding as in [8], we take a $P \in \mathcal{P}\left({ }^{k} E\right)$ with $\|P\|=1$, and a positive $\varepsilon$ such that $\varepsilon<1 / 3$. Since $E$ has the RNP, by [20, Theorem 14] there exists a $g \in E^{*}$ with $0<\|g\|<\varepsilon$ such that the function $|P|+g: B_{E} \rightarrow \mathbb{R}$ strongly attains its maximum; that is, there exists an $x_{0} \in B_{E}$ such that

$$
\left|P\left(x_{0}\right)\right|+g\left(x_{0}\right) \geq|P(x)|+g(x) \quad \forall x \in B_{E}
$$

and moreover, if $\lim _{n}\left(\left|P\left(x_{n}\right)\right|+g\left(x_{n}\right)\right)=\left|P\left(x_{0}\right)\right|+g\left(x_{0}\right)$ for a sequence in $B_{E}$, then necessarily $\lim _{n}\left\|x_{n}-x_{0}\right\|=0$. Observe that (1) is equivalent to

$$
\left|P\left(x_{0}\right)\right|+g\left(x_{0}\right) \geq|P(x)|+|g(x)| \quad \forall x \in B_{E} .
$$

It is easy to see that: $g\left(x_{0}\right) \geq 0$, by $(2) ;\left|P\left(x_{0}\right)\right|>0$, since $\|P\|=1$ and $\|g\|<1 / 3$; and $\left\|x_{0}\right\|=1$, since otherwise $\left|P\left(x_{0} /\left\|x_{0}\right\|\right)\right|+g\left(x_{0} /\left\|x_{0}\right\|\right)>$ $\left|P\left(x_{0}\right)\right|+g\left(x_{0}\right)$.

Let $f \in S_{E^{*}}$ be such that $f\left(x_{0}\right)=1$. Define $Q \in \mathcal{P}\left({ }^{k} E\right)$ by

$$
Q(x)=P(x)+g(x) f(x)^{k-1} \frac{P\left(x_{0}\right)}{\left|P\left(x_{0}\right)\right|} .
$$

We have $\|P-Q\| \leq\|g\|<\varepsilon$, and

$$
\left|Q\left(x_{0}\right)\right|=\left|P\left(x_{0}\right)\right|+g\left(x_{0}\right) \geq|P(x)|+|g(x)| \quad \forall x \in B_{E}
$$

and hence $\left|Q\left(x_{0}\right)\right| \geq|Q(x)|$ for every $x \in B_{E}$. 
On the other hand, if $\lim _{n}\left|Q\left(x_{n}\right)\right|=\left|Q\left(x_{0}\right)\right|$, then

$$
\lim _{n}\left|P\left(x_{n}\right)\right|+\left|g\left(x_{n}\right)\right|=\left|Q\left(x_{0}\right)\right|=\left|P\left(x_{0}\right)\right|+g\left(x_{0}\right)
$$

and defining $\varepsilon_{n}=\operatorname{sign}\left[g\left(x_{n}\right)\right]$, we have

$$
\lim _{n}\left(\left|P\left(\varepsilon_{n} x_{n}\right)\right|+g\left(\varepsilon_{n} x_{n}\right)\right)=\lim _{n}\left(\left|P\left(x_{n}\right)\right|+\left|g\left(x_{n}\right)\right|\right)=\left|P\left(x_{0}\right)\right|+g\left(x_{0}\right),
$$

hence $\lim _{n} \varepsilon_{n} x_{n}=x_{0}$ and consequently $Q$ strongly attains its norm.

In particular we see that the polynomials that strongly attain their norm form a second category set, provided that the Banach space has the RNP.

Observe also that if $E$ is a finite-dimensional space, then a polynomial strongly attains its norm if and only if it attains its norm at two points only, and that these polynomials form a $G_{\delta}$ dense subset of the space of all homogeneous polynomials.

In Section 4, we will see that many Banach spaces lack polynomials strongly attaining their norm. However, the following example shows that RNP is not necessary in order to have polynomials which attain their norm strongly.

ExAmple. The Banach space $E=l_{2} \oplus_{1} c_{0}$ does not have the RNP, since $\{0\} \oplus B_{c_{0}}$ is not dentable. The polynomial $P(x, y)=\sum_{n=1}^{\infty} n^{-1} e_{n}^{*}(x)^{2}$ strongly attains its norm since $\|P\|=1=P\left(e_{1}, 0\right)$ and if $\lim _{n} P\left(x_{n}, y_{n}\right)=1$ with $\left(x_{n}, y_{n}\right) \in S_{E}$, then necessarily $\left\{y_{n}\right\}$ converges to 0 , and $\left\{x_{n}\right\}$ to $e_{1}$.

The following easy local result points out where a polynomial can attain its norm strongly.

Proposition 2.7. Suppose that $P$ strongly attains its norm at a point $x_{0}$. If $P \in \mathcal{P}_{\mathrm{wb}}\left({ }^{k} E\right)$, then $i:\left(B_{E}, w\right) \rightarrow\left(B_{E},\|\|\right)$ is continuous at $x_{0}$; if $P \in \mathcal{P}_{\mathrm{wsc}}\left({ }^{k} E\right)$, then $i:\left(B_{E}, w\right) \rightarrow\left(B_{E},\|\|\right)$ is sequentially continuous at $x_{0}$.

If a linear functional strongly attains its norm at a point $x_{0}$, it is easy to see that $x_{0}$ is an extreme point. This result is no longer true in the homogeneous case if $k>1$. Indeed, $P:\left(\mathbb{R}^{2},\|\|_{\infty}\right) \rightarrow \mathbb{R}$ defined by $P(x, y)=$ $3 x^{2}-y^{2}$ strongly attains its norm at $(1,0)$ and $(-1,0)$, but neither $(1,0)$ nor $(-1,0)$ are extreme points.

\section{Gateaux differentiability}

Definition 3.1. We will say that a polynomial $P \in \mathcal{P}\left({ }^{k} E\right)$ polynomially approaches its norm whenever for any two sequences $\left\{x_{n}\right\}$ and $\left\{y_{n}\right\}$ in the unit sphere, satisfying $\lim _{n} P\left(x_{n}\right)=s_{x}\|P\|$ and $\lim _{n} P\left(y_{n}\right)=s_{y}\|P\|$, $s_{x}, s_{y} \in\{-1,+1\}$, we have $\lim _{n}\left(s_{x} Q\left(x_{n}\right)-s_{y} Q\left(y_{n}\right)\right)=0$ for every $Q \in$ $\mathcal{P}\left({ }^{k} E\right)$. 
As in Definition 2.1, if $k$ is even, then necessarily $s_{x} s_{y}=1$, more precisely: if $P$ polynomially approaches its norm, then there are no sequences in the unit sphere such that $\lim _{n} P\left(x_{n}\right)=\|P\|$ and $\lim _{n} P\left(y_{n}\right)=-\|P\|$. On the other hand, if $k$ is odd, then $P \in \mathcal{P}\left({ }^{k} E\right)$ polynomially approaches its norm if and only if for any sequences $\left\{x_{n}\right\}$ and $\left\{y_{n}\right\}$ in the unit sphere, satisfying $\lim _{n} P\left(x_{n}\right)=\|P\|=\lim _{n} P\left(y_{n}\right)$, we have $\lim _{n}\left(Q\left(x_{n}\right)-Q\left(y_{n}\right)\right)=0$ for every $Q \in \mathcal{P}\left({ }^{k} E\right)$.

Definition 3.2. We will say that $P$ weakly approaches its norm whenever there exists an $x_{0}^{* *} \in B_{E^{* *}}$ such that every sequence $\left\{x_{n}\right\} \subset S_{E}$ satisfying $\lim _{n}\left|P\left(x_{n}\right)\right|=\|P\|$ has a subsequence which is weak star convergent either to $x_{0}^{* *}$ or to $-x_{0}^{* *}$.

As above, every approaching sequence either is weak star convergent or may be split in two subsequences $\left\{x_{n}^{+}\right\}$and $\left\{x_{n}^{-}\right\}$converging, in the weak star topology, to $x_{0}^{* *}$ and $-x_{0}^{* *}$ respectively.

Observe that if a polynomial attains its norm and approaches it weakly, then it attains its norm exactly at two points. The next proposition says us something more.

Proposition 3.3. If $P \in \mathcal{P}\left({ }^{k} E\right)$ weakly approaches its norm and $\widetilde{P}$ attains its norm at a point $x_{0}^{* *} \in S_{E^{* *}}$, then every sequence $\left\{x_{n}^{* *}\right\} \subset B_{E^{* *}}$ such that $\lim _{n}\left|\widetilde{P}\left(x_{n}^{* *}\right)\right|=\|\widetilde{P}\|$ has a subsequence which is weak star convergent either to $x_{0}^{* *}$ or to $-x_{0}^{* *}$.

Proof. Suppose that there is a sequence $\left\{z_{n}^{* *}\right\} \subset B_{E^{* *}}$ for which $\lim _{n} \widetilde{P}\left(z_{n}^{* *}\right)=\|\widetilde{P}\|$ and which has a weak star cluster point $z_{0}^{* *}$ such that $z_{0}^{* *}-x_{0}^{* *} \neq 0$ and $z_{0}^{* *}+x_{0}^{* *} \neq 0$. If $z_{0}^{* *}$ and $x_{0}^{* *}$ are linearly independent, we may choose an $f \in S_{E^{*}}$ such that $f\left(x_{0}^{* *}\right)=0$ and $f\left(z_{0}^{* *}\right)=\alpha>0$. Now, if we define

$$
W=\left\{z^{* *} \in E^{* *}:\left|f\left(z^{* *}\right)\right|<\alpha / 3\right\}
$$

we have $W \cap\left(z_{0}^{* *}+W\right)=\emptyset$, and since $W$ and $z_{0}^{* *}+W$ are neighborhoods of $x_{0}^{* *}$ and $z_{0}^{* *}$ respectively, we may assume that for every $n, z_{n}^{* *} \in z_{0}^{* *}+W$, and $x_{n} \in W$ where $\left\{x_{n}\right\} \subset S_{E}$ is an approaching sequence drawn from the net provided by Davie-Gamelin's Theorem (see [9, Theorem 1]). For every $n$, we may choose a $z_{n} \in B_{E} \cap\left(z_{0}^{* *}+W\right)$ such that $P\left(z_{n}\right)>\widetilde{P}\left(z_{n}^{* *}\right)-1 / n$ by [9] again. So, for every $n$, we have

$$
\left|f\left(z_{n}\right)\right|>2 \alpha / 3>\alpha / 3>\left|f\left(x_{n}\right)\right| .
$$

These inequalities also hold if we normalize the sequence $\left\{z_{n}\right\}$, hence we have two approaching sequences such that

$$
|| f\left(z_{n}\right)|-| f\left(x_{n}\right)||>\alpha / 3
$$


and therefore $P$ does not approach its norm weakly. The same kind of argument works if $x_{0}^{* *}$ and $z_{0}^{* *}$ are linearly dependent. It is enough to choose an $f \in S_{E^{*}}$ such that $f\left(x_{0}^{* *}-z_{0}^{* *}\right) \neq 0$.

Following [7], we will say that a sequence $\left\{x_{n}\right\}$ is $\left(\mathcal{P}_{k}\right)$-Cauchy provided that $\left\{Q\left(x_{n}\right)\right\}$ converges for every $Q \in \mathcal{P}\left({ }^{k} E\right)$. If $\lim _{n} Q\left(x_{n}\right)=Q(x)$ for every $Q \in \mathcal{P}\left({ }^{k} E\right)$, we will say that $\left\{x_{n}\right\}$ is $\left(\mathcal{P}_{k}\right)$-convergent to $x$.

The next proposition follows immediately from the definitions.

Proposition 3.4. $P \in \mathcal{P}\left({ }^{k} E\right)$ polynomially approaches its norm if and only if every sequence $\left\{x_{n}\right\} \subset S_{E}$ such that $\lim _{n} P\left(x_{n}\right)= \pm\|P\|$ is $\left(\mathcal{P}_{k}\right)$ Cauchy.

Next we find the relationship between Definitions 3.1 and 3.2: as one could expect, to approach the norm polynomially is stronger than to do it weakly.

Proposition 3.5. If $P \in \mathcal{P}\left({ }^{k} E\right)$ polynomially approaches its norm, then it approaches it weakly.

Proof. Let $\left\{x_{n}\right\}$ be an approaching sequence. First, we prove that $\left\{x_{n}\right\}$ has a weakly Cauchy subsequence, and consequently a weak star convergent subsequence. Passing to a subsequence we may assume that $\lim _{n} P\left(x_{n}\right)=$ $\pm\|P\|$, hence $\left\{x_{n}\right\}$ is $\left(\mathcal{P}_{k}\right)$-Cauchy.

We may assume that $\left\{x_{n}\right\}$ is not weakly null, hence there exists a subsequence, still denoted by $\left\{x_{n}\right\}$, and an $f \in S_{E^{*}}$ such that $\lim _{n} f\left(x_{n}\right)=$ $\alpha>0$. If $\left\{x_{n}\right\}$ is weakly Cauchy, then we are done; otherwise there exists a $g \in S_{E^{*}}$ such that $\lim _{n} g\left(x_{n}\right)$ does not exist. For the polynomial $Q=f^{k-1} g$, $\lim _{n} Q\left(x_{n}\right)$ does not exist, contradicting the fact that $\left\{x_{n}\right\}$ is $\left(\mathcal{P}_{k}\right)$-Cauchy. Denote the limit of such a subsequence by $x_{0}^{* *}, x_{0}^{* *} \in B_{E^{* * *}}$.

In order to prove that $P$ weakly approaches its norm, we have to check that every approaching sequence $\left\{x_{n}\right\}$ has a subsequence converging, in the weak star topology, either to $x_{0}^{* *}$ or to $-x_{0}^{* *}$. As usual, we may assume that $\lim _{n} P\left(x_{n}\right)= \pm\|P\|$. Take a weak star cluster point $y_{0}^{* *}$ different from $x_{0}^{* *}$ and $-x_{0}^{* *}$. If $x_{0}^{* *}$ and $y_{0}^{* *}$ are linearly dependent, suppose that $\left\|x_{0}^{* *}-y_{0}^{* *}\right\| \leq$ $\left\|x_{0}^{* *}+y_{0}^{* *}\right\|$ and take an $f \in S_{E^{*}}$ such that

$$
\left|f\left(x_{0}^{* *}+y_{0}^{* *}\right)\right| \geq\left|f\left(x_{0}^{* *}-y_{0}^{* *}\right)\right|>a
$$

for some $a>0$. Defining $W$ as $\left\{x^{* *} \in X:\left|f\left(x^{* *}\right)\right|<a / 3\right\}$, we have

$$
\left|f\left(x^{* *}+y^{* *}\right)\right|>a / 3 \text { and }\left|f\left(x^{* *}-y^{* *}\right)\right|>a / 3
$$

provided that $x^{* *} \in x_{0}^{* *}+W$ and $y^{* *} \in y_{0}^{* *}+W$. This gives $\mid f\left(x^{* *}\right)^{k}-$ $f\left(y^{* *}\right)^{k} \mid>2(a / 6)^{k}$ if $k$ is odd, and $\left|f\left(x^{* *}\right)^{k}-f\left(y^{* *}\right)^{k}\right|>(a / 3)^{k}$ if $k$ is even. Now, the fact that both $x_{0}^{* *}+W$ and $y_{0}^{* *}+W$ contain an infinite number of terms of the original sequence allows us to define two subsequences of $\left\{x_{n}\right\}$, 
$\left\{x_{n_{i}}\right\}$ and $\left\{x_{m_{i}}\right\}$, such that

$$
Q\left(x_{n_{i}}\right)-Q\left(x_{m_{i}}\right)>(a / 3)^{k} 2^{1-k}
$$

for $Q=f^{k}$, which contradicts the fact that $P$ polynomially approaches its norm.

On the other hand, if $x^{* *}$ and $y^{* *}$ are linearly independent, we may choose $f \in S_{E^{*}}$ such that $f\left(x_{0}^{* *}\right)=0$ and $f\left(y_{0}^{* *}\right) \neq 0$, and proceed as above.

However both definitions are not equivalent, as the following example shows.

Example. Define $P \in \mathcal{P}\left({ }^{2} l_{2}\right)$ by

$$
P(x)=\sum_{n=1}^{\infty}\left(1-\frac{1}{n+1}\right) e_{n}^{*}(x)^{2} .
$$

We have $0 \leq P(x)<\|x\|^{2}$, and $P(x)=0$ if and only if $x=0$. Moreover $\|P\|=1$ since $P\left(e_{n}\right)=1-1 /(n+1)$.

If $\left\{x_{n}\right\} \subset S_{E}$ satisfies $\lim _{n} P\left(x_{n}\right)=1$, than

$$
\lim _{n} \sum_{i=1}^{\infty} \frac{1}{i+1} e_{i}^{*}\left(x_{n}\right)^{2}=0,
$$

hence $\lim _{n} e_{i}^{*}\left(x_{n}\right)=0$ for every $i$, and consequently $\left\{x_{n}\right\}$ is weakly null. Therefore $P$ weakly attains its norm.

On the other hand, if we define $Q(x)=\sum_{n=1}^{\infty} e_{2 n}^{*}(x)^{2}$, then $\left\{Q\left(e_{n}\right)\right\}$ does not converge, and therefore $P$ does not approach its norm polynomially.

The polynomial in the example above does not belong to $\mathcal{P}_{\text {wsc }}\left({ }^{2} l_{2}\right)=$ $\mathcal{P}_{\mathrm{c}}\left({ }^{2} l_{2}\right)$. It also proves that in general the Aron-Berner extension of a polynomial does not attain its norm, even if the polynomial weakly approaches it.

We are looking for conditions on the Banach space which allow us to prove the converse of Proposition 3.5. Recall that a Banach space has the Dunford-Pettis Property (DPP) whenever given weakly null sequences, $\left\{x_{n}\right\}$ and $\left\{f_{n}\right\}$, in $E$ and $E^{*}$ respectively, then $\lim _{n} f_{n}\left(x_{n}\right)=0 . C(K)$ and $L^{1}(\mu)$ are examples of spaces that have the DPP.

If the Banach space $E$ has the DPP, then $\mathcal{P}_{\text {wsc }}\left({ }^{k} E\right)=\mathcal{P}\left({ }^{k} E\right)$ (see [19]). However, as the Banach space $l_{1}$ shows, in general it is not true that DPP implies $\mathcal{P}_{\mathrm{c}}\left({ }^{k} E\right)=\mathcal{P}\left({ }^{k} E\right)$. On the other hand there are Banach spaces, the Tsirelson space $T^{*}$ for example, that do not have the DPP but satisfy $\mathcal{P}_{\mathrm{c}}\left({ }^{k} E\right)=\mathcal{P}\left({ }^{k} E\right)([2])$. We are going to use these properties in order to get the equivalence between approaching the norm polynomially and weakly.

Proposition 3.6. If $E$ either has the DPP or satisfies $\mathcal{P}_{\mathrm{c}}\left({ }^{k} E\right)=$ $\mathcal{P}\left({ }^{k} E\right)$, then $P$ approaches its norm polynomially if and only if it does weakly. 
Proof. We prove only the even case. We start with two sequences $\left\{x_{n}\right\}$ and $\left\{y_{n}\right\}$ in the unit sphere such that

$$
\lim _{n}\left|P\left(x_{n}\right)\right|=\lim _{n}\left|P\left(y_{n}\right)\right|=\|P\| .
$$

We may split the sequences into $\left\{x_{n}^{+}\right\}$and $\left\{y_{n}^{+}\right\}$weak star convergent to $x_{0}^{* *}$, and $\left\{x_{n}^{-}\right\}$and $\left\{y_{n}^{-}\right\}$weak star convergent to $-x_{0}^{* *}$, since $P$ approaches its norm weakly. Consequently, there exist $\varepsilon_{x}, \varepsilon_{y}: \mathbb{N} \rightarrow\{-1,+1\}$ such that both $\left\{\varepsilon_{x}(n) x_{n}\right\}$ and $\left\{\varepsilon_{y}(n) y_{n}\right\}$ converge to $x_{0}^{* *}$. If $E$ has the DPP, we consider the sequence $\left\{z_{n}\right\}$ defined by

$$
z_{2 n-1}=\varepsilon_{x}(n) x_{n} \quad \text { and } \quad z_{2 n}=\varepsilon_{y}(n) y_{n} .
$$

It is weakly Cauchy, hence, by [19], $\left\{Q\left(z_{n}\right)\right\}$ converges for every $Q \in \mathcal{P}\left({ }^{k} E\right)$, in particular $\lim _{n} P\left(x_{n}\right)=\lim _{n} P\left(y_{n}\right)$, and consequently

$$
\lim _{n}\left(Q\left(x_{n}\right)-Q\left(y_{n}\right)\right)=\lim _{n}\left(Q\left(\varepsilon_{x}(n) x_{n}\right)-Q\left(\varepsilon_{y}(n) y_{n}\right)\right)=0 .
$$

On the other hand, if $\mathcal{P}_{\mathrm{c}}\left({ }^{k} E\right)=\mathcal{P}\left({ }^{k} E\right)$, then for every $Q \in \mathcal{P}\left({ }^{k} E\right)=\mathcal{P}_{\mathrm{c}}\left({ }^{k} E\right)$, from Lemma 1.2 we have

$$
\widetilde{Q}\left(x_{0}^{* *}\right)=\widetilde{Q}\left(-x_{0}^{* *}\right)=\lim _{n} Q\left(x_{n}\right)=\lim _{n} Q\left(y_{n}\right),
$$

in particular $\lim _{n} P\left(x_{n}\right)=\lim _{n} P\left(y_{n}\right)$, hence $\lim _{n}\left(Q\left(x_{n}\right)-Q\left(y_{n}\right)\right)=0$.

The following theorem is the analogue of Theorem 2.4 for Gateaux differentiability, and motivates the introduction of the concept of polynomial approach.

Theorem 3.7. $P \in \mathcal{P}\left({ }^{k} E\right)$ polynomially approaches its norm if and only if \|\|$: \mathcal{P}\left({ }^{k} E\right) \rightarrow \mathbb{R}$ is Gateaux differentiable at $P$.

Proof. Assume that the norm is Gateaux differentiable at $P$. We may assume that $\|P\|=1$. Take two sequences as in Definition 3.1, and fix $Q \in S_{\mathcal{P}\left({ }^{k} E\right)}$. As the norm is Gateaux differentiable at $P$, for every $\varepsilon$ there exists a $\delta$ such that if $|t|<\delta$ then

$$
\|P+t Q\|+\|P-t Q\| \leq 2+\varepsilon|t|,
$$

therefore

$$
\left|(P+t Q)\left(x_{n}\right)\right|+\left|(P-t Q)\left(y_{n}\right)\right| \leq 2+\varepsilon|t| .
$$

If $\delta$ is small enough we have

$$
\left|(P+t Q)\left(x_{n}\right)\right|=s_{x}(P+t Q)\left(x_{n}\right) \quad \text { and } \quad\left|(P-t Q)\left(y_{n}\right)\right|=s_{y}(P-t Q)\left(y_{n}\right),
$$

hence

$$
\begin{aligned}
s_{x} P\left(x_{n}\right)+s_{y} P\left(y_{n}\right)+ & \left(s_{x} t Q\left(x_{n}\right)-s_{y} t Q\left(y_{n}\right)\right) \\
& =s_{x}(P+t Q)\left(x_{n}\right)+s_{y}(P-t Q)\left(y_{n}\right) \leq 2+\varepsilon|t| .
\end{aligned}
$$


From $\lim _{n}\left(s_{x} P\left(x_{n}\right)+s_{y} P\left(y_{n}\right)\right)=2$, it follows that there exists an $n_{0}$ such that if $n>n_{0}$ then $2-\left(s_{x} P\left(x_{n}\right)+s_{y} P\left(y_{n}\right)\right)<\varepsilon \delta$, and consequently

$$
t\left(s_{x} Q\left(x_{n}\right)-s_{y} Q\left(y_{n}\right)\right) \leq 2 \varepsilon \delta \quad \forall n>n_{0} \forall t \text { with }|t|<\delta,
$$

or equivalently $\lim _{n}\left(s_{x} Q\left(x_{n}\right)-s_{y} Q\left(y_{n}\right)\right)=0$. So $P$ polynomially approaches its norm.

We omit the proof of the converse which is similar to that of Theorem 2.4 modified as above.

REMARK. If we denote by $G^{\prime}(P)$ the Gateaux differential of the norm at $P$, it is easy to see that

$$
G^{\prime}(P)(Q)=\lim _{n} Q\left(x_{n}\right)
$$

where $\left\{x_{n}\right\}$ is any sequence in the unit sphere such that $\lim _{n} P\left(x_{n}\right)=\|P\|$. Indeed, observe first that $G^{\prime}(P)$ does not depend on the choice of $\left\{x_{n}\right\}$ because of Proposition 3.4. On the other hand, if there is no such sequence (which is possible if $k$ is even) it is enough to realize that $G^{\prime}(P)=-G^{\prime}(-P)$. Let us prove (1):

We have $t Q\left(x_{n}\right) \leq\|P+t Q\|-P\left(x_{n}\right)$ since $P\left(x_{n}\right)+t Q\left(x_{n}\right) \leq\|P+t Q\|$. Hence

$$
Q\left(x_{n}\right) \leq \frac{\|P+t Q\|-P\left(x_{n}\right)}{t} \text { and } \quad Q\left(x_{n}\right) \geq \frac{\|P+t Q\|-P\left(x_{n}\right)}{t}
$$

for $t>0$ and $t<0$ respectively. Passing to the limit we get

$$
\lim _{n} Q\left(x_{n}\right) \leq \frac{\|P+t Q\|-\|P\|}{t} \text { and } \lim _{n} Q\left(x_{n}\right) \geq \frac{\|P+t Q\|-\|P\|}{t},
$$

and therefore

$$
\begin{aligned}
G^{\prime}(P)(Q) & =\lim _{t \rightarrow 0^{-}} \frac{\|P+t Q\|-\|P\|}{t} \leq \lim _{n} Q\left(x_{n}\right) \\
& \leq \lim _{t \rightarrow 0^{+}} \frac{\|P+t Q\|-\|P\|}{t}=G^{\prime}(P)(Q) .
\end{aligned}
$$

The following corollaries are easy consequences of Theorem 3.7.

Corollary 3.8. If $P \in \mathcal{P}\left({ }^{k} E\right)$ attains its norm and \|\|$: \mathcal{P}\left({ }^{k} E\right) \rightarrow \mathbb{R}$ is Gateaux differentiable at $P$, then it attains its norm exactly at two points.

The example below shows that the converse of this corollary is not true. Even approaching the norm weakly is not necessary in order to attain it only at two points.

ExAmple. The polynomial $P \in \mathcal{P}\left({ }^{k} l_{1}\right)$ given by

$$
P(x)=e_{1}^{*}(x)^{k}+\sum_{n=2}^{\infty} \frac{n-1}{n} e_{n}^{*}(x)^{k}
$$


attains its norm only at $\pm e_{1}$, but does not approach its norm weakly since $\lim _{n} P\left(e_{n}\right)=\|P\|$ and $\left\{e_{n}\right\}$ does not converge weakly to $e_{1}$.

Corollary 3.9. If $P \in \mathcal{P}\left({ }^{k} E\right)$ attains its norm at $x_{0}$ and \|\|$: \mathcal{P}\left({ }^{k} E\right)$ $\rightarrow \mathbb{R}$ is Gateaux differentiable at $P$, then every sequence $\left\{x_{n}\right\}$ such that $\lim _{n} P\left(x_{n}\right)= \pm\|P\|$ is $\left(\mathcal{P}_{k}\right)$-convergent to $\pm x_{0}$.

Proof. Suppose that $\|P\|=P\left(x_{0}\right)$. The sequence $\left\{z_{n}\right\}$ defined by $z_{2 n}=$ $x_{n}$ and $z_{2 n+1}=x_{0}$ is $\left(\mathcal{P}_{k}\right)$-Cauchy, and consequently $\lim _{n} Q\left(x_{n}\right)=Q\left(x_{0}\right)$ for every $Q \in \mathcal{P}\left({ }^{k} E\right)$.

COROLlary 3.10. Let $E$ be a Banach space with the property that every sequence $\left\{x_{n}\right\} \subset S_{E}$ weakly convergent to an $x_{0} \in S_{E}$ is norm convergent. Let $P \in \mathcal{P}\left({ }^{k} E\right)$ attain its norm. Then the following are equivalent:

(i) The norm is Fréchet differentiable at $P$.

(ii) The norm is Gateaux differentiable at $P$.

(iii) $P$ approaches its norm weakly.

Proof. If $\|P\|=\left|P\left(x_{0}\right)\right|$, then every approaching sequence may be split in two subsequences weakly convergent to $x_{0}$ and $-x_{0}$ respectively, since $P$ approaches its norm weakly. Both sequences are norm convergent, and we get the result.

Schur spaces, such as $l_{1}$ and spaces whose norms have the Kadec-Klee property, satisfy the required condition. The norms of uniformly convex spaces, e.g. $L_{p}(\mu), 1<p<\infty$, have the Kadec-Klee property.

Of course to attain the norm only at two points does not guarantee that it is strongly attained. The polynomial $P \in \mathcal{P}\left({ }^{2} l_{2}\right)$ given by

$$
P(x)=e_{1}^{*}(x)^{2}+\sum_{n=2}^{\infty}(1-1 / n) e_{n}^{*}(x)^{2}
$$

satisfies $1=\|P\|=P\left(e_{1}\right),|P(x)|<1$ if $x \neq \pm e_{1}$, and $\lim _{n} P\left(e_{n}\right)=1$.

The following example proves that, in general, it is not true that Fréchet and Gateaux differentiability are equivalent if $P$ attains its norm.

Example. $P \in \mathcal{P}\left({ }^{2} c_{0}\right)$ defined by

$$
P(x)=e_{1}^{*}(x)^{2}-\frac{1}{4} \sum_{n=2}^{\infty} \frac{1}{2^{n}} e_{n}^{*}(x)^{2}
$$

attains its norm at $\pm e_{1}$ (and only there). It approaches its norm weakly, and by Proposition 3.6 and Theorem 3.7, the norm is Gateaux differentiable at $P$. But $P$ does not attain its norm strongly since $\lim _{n} P\left(e_{1}+e_{n}\right)=\|P\|$. 
However, we have the following local result. Recall that a point $x_{0} \in S_{E}$ is a denting point if for every positive $\varepsilon$,

$$
x_{0} \notin \overline{\mathrm{CO}}\left(B_{E}-B\left(x_{0}, \varepsilon\right)\right) .
$$

Proposition 3.11. If $P \in \mathcal{P}\left({ }^{k} E\right)$ attains its norm at a point at which the identity $i:\left(B_{E}, w\right) \rightarrow\left(B_{E},\|\|\right)$ is sequentially continuous, in particular at a denting point, then the norm is Fréchet differentiable at $P$ if and only if $P$ approaches its norm weakly.

Proof. Let $x_{0} \in S_{E}$ be a denting point such that $\|P\|=\left|P\left(x_{0}\right)\right|$. It is enough to observe that if a sequence $\left\{x_{n}\right\} \subset S_{E}$ is weakly convergent to $x_{0}$, then it is norm convergent, because otherwise, we may assume that there exists an $\varepsilon>0$ such that $\left\{x_{n}\right\} \subset B_{E}-B\left(x_{0}, \varepsilon\right)$, and realizing that $x_{0}$ belongs to the weak closure of the range of $\left\{x_{n}\right\}$, we find that $x_{0}$ belongs to the weak closure of $\operatorname{co}\left(B_{E}-B\left(x_{0}, \varepsilon\right)\right)$, that is, $x_{0} \in \overline{\mathrm{co}}\left(B_{E}-B\left(x_{0}, \varepsilon\right)\right)$, which is a contradiction because $x_{0}$ is a denting point.

The fact that $\mathcal{P}\left({ }^{k} E\right)$ is a separable space, for $k>1$, is a very strong condition; in fact, as $E^{*}$ is a subspace of $\mathcal{P}\left({ }^{k} E\right)$ for every $k$, it implies that $E^{*}$ is separable. However, the converse is not true. For example $\mathcal{P}\left({ }^{k} l_{k}\right)$, $k>1$, contains $l_{\infty}$ as a subspace, hence it is not separable (see [13]). On the other hand, $\mathcal{P}\left({ }^{k} c_{0}\right)$ is separable for every $k$, and it has a basis.

Corollary 3.12. If $\mathcal{P}\left({ }^{k} E\right)$ is separable then the set of all polynomials which polynomially approach their norm is a $G_{\delta}$ dense subset of $\mathcal{P}\left({ }^{k} E\right)$.

Proof. Observe that $\mathcal{P}\left({ }^{k} E\right)$ is weakly Asplund and hence the norm is Gateaux differentiable in a $G_{\delta}$ dense set (see [10]).

4. Some examples. In this section we study the concepts introduced above for some classical Banach spaces. We start by considering the Banach space $\left(C(K),\|\|_{\infty}\right)$ where $K$ is a Hausdorff compact space. It is well known that a bounded sequence $\left\{x_{n}\right\}$ converges weakly to $x_{0}$ if and only if it converges pointwise (see [11, p. 86]). From this we deduce the following result.

Proposition 4.1. Let $K$ be an infinite Hausdorff compact space. Then \|\|$: \mathcal{P}\left({ }^{k} C(K)\right) \rightarrow \mathbb{R}$ is nowhere Fréchet differentiable.

Proof. We claim that given $x_{0} \in S_{C(K)}$ there exists a sequence $\left\{x_{n}\right\} \subset$ $B_{C(K)}$ which is weakly convergent to $x_{0}$, but does not converge in norm. Every $P \in \mathcal{P}\left({ }^{k} C(K)\right)$ is weakly sequentially continuous since $C(K)$ has the DPP, hence it cannot attain its norm strongly at $x_{0} \in S_{C(K)}$.

Now we prove the claim. Let $\left\{y_{n}\right\}$ be a sequence of continuous functions such that $y_{n}: K \rightarrow[0,1 / 2],\left\|y_{n}\right\|_{\infty}=y\left(t_{n}\right)=1 / 2$ and $\left\{y_{n}\right\}$ converges pointwise to 0 ; such a sequence exists because $C(K)$ is not Schur. Define 
$z_{n}$ as $x_{0}-y_{n}$ if $x_{0}\left(t_{n}\right)>1 / 2$, and as $x_{0}+y_{n}$ otherwise. The functions $x_{n}=\varphi \circ z_{n}$, where $\varphi(t)=t$ if $|t| \leq 1$ and 1 otherwise, fulfil the required conditions.

We now consider the Banach space $c_{0}$. It is easy to see directly that a $P \in \mathcal{P}\left({ }^{k} c_{0}\right)$ never attains its norm strongly, since if $P$ attains its norm at $x_{0}$, then the sequence $\left\{x_{0}+\left(1-e_{n}^{*}\left(x_{0}\right)\right) e_{n}\right\}$ converges weakly to $x_{0}$, is eventually contained in $B_{c_{0}}$ and is not norm convergent to $x_{0}$. On the other hand, $\lim _{n} P\left(x_{0}+\left(1-e_{n}^{*}\left(x_{0}\right)\right) e_{n}\right)=P\left(x_{0}\right)$.

We know also, by Proposition 1.3, that $\widetilde{P}$ attains its norm at a point $x^{* *} \in B_{l_{\infty}}$ for every $P \in \mathcal{P}\left({ }^{k} c_{0}\right)$. Moreover, by Proposition 3.6 and Theorem 3.7, a polynomial $P$ weakly approaches its norm if and only if the norm is Gateaux differentiable at $P$. We may say something more.

Proposition 4.2. The norm of $\mathcal{P}\left({ }^{k} c_{0}\right)$ is Gateaux differentiable at $P$ if and only if $\widetilde{P}$ attains its norm at two points only.

Proof. We have to prove the "if" part only. If the norm is not Gateaux differentiable, there are approaching sequences converging, in the weak star topology, to points $x_{0}^{* *} \neq \pm y_{0}^{* *}$, since the weak topology is metrizable on the unit ball. Hence $\widetilde{P}$ attains its norm at $\pm x_{0}$ and $\pm y_{0}$.

Now, by Corollary 3.12, the set of polynomials which approach weakly its norm is a $G_{\delta}$ dense subset of $\mathcal{P}\left({ }^{k} c_{0}\right)$. In particular, all the polynomials $P$ but a first category subset have the property that $\widetilde{P}$ attains its norm exactly at two points. Therefore the set of polynomials which attain their norm at more than two points is a first category set. In fact, if we denote by $\mathcal{N} \mathcal{A P}\left({ }^{k} c_{0}\right)$ the set of all norm attaining polynomials, then $\mathcal{N} \mathcal{A} \mathcal{P}\left({ }^{k} c_{0}\right)$ is a first category subset of $\mathcal{P}\left({ }^{k} c_{0}\right)$, but before proving it we must introduce some notation.

It is easy to see that if $Q_{n}=(1 / k !) d^{k-1} P\left(e_{n}\right)$, then

$$
P(x)=\sum_{n=1}^{\infty} e_{n}^{*}(x) Q_{n}(x) .
$$

Lemma 4.3. Let $x \in c_{0}$ be such that $\|P\|=P(x)$. Then $Q_{n}(x)=0$ for every $n$ such that $\left|e_{n}^{*}(x)\right|<1$.

Proof. Define $\phi_{n}(t)=P\left(x+t e_{n}\right)$ for such $n$ 's. Then $k Q_{n}(x)=\phi_{n}^{\prime}(0)$, because

$$
\begin{aligned}
\phi_{n}(t)= & P(x)+\left(\begin{array}{c}
k \\
1
\end{array}\right) \widehat{P}\left(x, \ldots, x, e_{n}\right) t+\ldots+ \\
& +\left(\begin{array}{c}
k \\
k-1
\end{array}\right) \widehat{P}\left(x, e_{n}, \ldots, e_{n}\right) t^{k-1}+P\left(e_{n}\right) t^{k}
\end{aligned}
$$

and $\phi_{n}^{\prime}(0)=0$ because $x+t e_{n} \in S_{c_{0}}$ if $t$ is small enough. 
THEOREM 4.4. $\mathcal{N} \mathcal{A P}\left({ }^{k} c_{0}\right)$ is a first category subset of $\mathcal{P}\left({ }^{k} c_{0}\right)$.

Proof. We define $\mathcal{A}_{N}$ and $\mathcal{B}_{N}$ as

$\mathcal{A}_{N}=\left\{P: \exists x \in S_{c_{0}}\right.$ such that $P(x)=\|P\|$ and $\left.\left|e_{n}^{*}(x)\right|<1 \forall n>N\right\}$,

$\mathcal{B}_{N}=\left\{P:\left\{Q_{j}\right\}_{j}\right.$ satisfies $\left.\bigcap_{j>N} V\left(\widetilde{Q}_{j}\right) \neq\{0\}\right\}$,

where $\widetilde{Q}_{j}$ is the Aron-Berner extension to $l_{\infty}$ of $Q_{j}, V\left(\widetilde{Q}_{j}\right) \subset l_{\infty}$ is the set of zeros of $\widetilde{Q}_{j}$, and $\left\{Q_{j}\right\}_{j}$ is the associated sequence that we defined in (1). Clearly $\mathcal{N} \mathcal{A} \mathcal{P}\left({ }^{k} c_{0}\right)=\bigcup_{N=1}^{\infty}\left(\mathcal{A}_{N} \cup\left(-\mathcal{A}_{N}\right)\right)$ and $\mathcal{A}_{N} \subset \mathcal{B}_{N}$ because if $\|P\|=P(x)$ then $x \in V\left(Q_{j}\right)$ for every $j>N$ by the lemma above.

Our goal is to prove that $\mathcal{A}_{N}$ is nowhere dense for every positive integer $N$. We start by proving that $\overline{\mathcal{A}}_{N} \subset \mathcal{B}_{N}$.

Let $\left\{P_{n}\right\} \subset \mathcal{A}_{N}$ and $P_{n} \stackrel{\|}{\rightarrow} P$. Observe first that if $\left\{Q_{j}^{n}\right\}_{j}$ and $\left\{Q_{j}\right\}_{j}$ are the sequences associated with $P_{n}$ and $P$ respectively by (1), then $Q_{j}^{n} \stackrel{\|}{\rightarrow} Q_{j}$. Moreover the convergence is uniform with respect to $j$, because

$$
\left|Q_{j}(y)-Q_{j}^{n}(y)\right|=\left|\widehat{P}\left(e_{j}, y, \ldots, y\right)-\widehat{P}_{n}\left(e_{j}, y, \ldots, y\right)\right| \leq\left\|\widehat{P}-\widehat{P}_{n}\right\| \cdot\|y\|^{k-1} .
$$

In order to have $P \in \mathcal{B}_{N}$ we need to check that $\bigcap_{j>N} V\left(\widetilde{Q}_{j}\right) \neq\{0\}$.

Choose $x_{n} \in S_{c_{0}}$ such that $P_{n}\left(x_{n}\right)=\left\|P_{n}\right\|$ and $\left|e_{j}^{*}\left(x_{n}\right)\right|<1$ for every $j>N$. By Lemma 4.3 we have

$$
Q_{j}^{n}\left(x_{n}\right)=0 \quad \forall j>N \forall n \in \mathbb{Z}^{+} .
$$

The sequence $\left\{x_{n}\right\}$ (strictly speaking, a subsequence) is $w^{*}$-convergent to $x_{0} \in B_{l_{\infty}}$ because $\sigma\left(l_{\infty}, l_{1}\right)$ is metrizable on bounded sets. Now

$$
\left|\widetilde{Q}_{j}\left(x_{0}\right)-Q_{j}^{n}\left(x_{n}\right)\right| \leq\left|\widetilde{Q}_{j}\left(x_{0}\right)-Q_{j}\left(x_{n}\right)\right|+\left|Q_{j}\left(x_{n}\right)-Q_{j}^{n}\left(x_{n}\right)\right|
$$

and hence by the uniform convergence of $\left\{Q_{j}^{n}\right\}$ to $Q_{j}$ and the $w^{*}$-continuity of $\widetilde{Q}_{j}$ on $B_{l_{\infty}}$, we see that $\widetilde{Q}_{j}\left(x_{0}\right)=\lim _{n} Q_{j}^{n}\left(x_{n}\right)$ for all $j$ and therefore $x_{n} \in \bigcap_{j>N} V\left(\widetilde{Q}_{j}\right)$. But $x_{0} \neq 0$ unless $P \equiv 0$ because

$$
\|P\|=\lim _{n}\left\|P_{n}\right\|=\lim _{n} P_{n}\left(x_{n}\right)=\lim _{n} \widetilde{P}_{n}\left(x_{n}\right)=\widetilde{P}\left(x_{0}\right) .
$$

So $P$ belongs to $\mathcal{B}_{N}$ because the identically 0 polynomial also belongs to $\mathcal{B}_{N}$.

The final step is to prove that $\operatorname{Int}\left(\mathcal{B}_{N}\right)=\emptyset$.

Given any ball $B(P, \varepsilon) \subset \mathcal{P}\left({ }^{k} c_{0}\right)$, there exists an $F \in B(P, \varepsilon / 2)$ which only depends on a finite number of coordinates $e_{1}^{*}(x), \ldots, e_{n}^{*}(x)$ (see [3]). We may suppose that $n>N$. Set

$G(x)=F(x)+\frac{\varepsilon}{2}\left(\frac{1}{2^{n+1}} e_{n+1}^{*}(x)\left(e_{1}^{*}(x)\right)^{k-1}+\ldots+\frac{1}{2^{j}} e_{j}^{*}(x)\left(e_{j-n}^{*}(x)\right)^{k-1}+\ldots\right)$.

Then $G \in B(P, \varepsilon)$ because $\|G-F\| \leq \varepsilon / 2$. 
The $\left\{Q_{j}\right\}_{j>n}$ corresponding to $G$ are

$$
\frac{\varepsilon}{2}\left(\frac{1}{2^{n+j}} \cdot \frac{k-1}{k} e_{n+j}^{*}(x)\left(e_{j}^{*}(x)\right)^{k-2}+\frac{1}{2^{j}} \cdot \frac{1}{k}\left(e_{j-n}^{*}(x)\right)^{k-1}\right) .
$$

The functional $\widetilde{Q}_{j}: l_{\infty} \rightarrow \mathbb{R}$ is defined similarly and

$$
\bigcap_{j>N} V\left(\widetilde{Q}_{j}\right) \subset \bigcap_{j>n} V\left(\widetilde{Q}_{j}\right)=\{0\} .
$$

So $G \notin \mathcal{B}_{N}$ and the proof is finished.

It is an open question whether this result depends on the degree $k$ for any Banach space.

We now consider the Banach spaces $\left(L_{1}(\mu),\|\|_{1}\right)$. We know that if a polynomial attains its norm and approaches it weakly, then it attains the norm only at two points. For the Banach spaces $L_{1}(\mu)$ we have a stronger result.

Proposition 4.5. If $P \in \mathcal{P}\left({ }^{k} L_{1}(\mu)\right)$ approaches its norm weakly, then $P$ attains it only at two points.

Proof. It is enough to realize that every polynomial on $L_{1}(\mu)$ is weakly sequentially continuous (since $L_{1}(\mu)$ has the DPP), and that weakly Cauchy and weakly convergent sequences in $L_{1}(\mu)$ coincide [11, p. 86].

The converse of this proposition is not true: the example following Corollary 3.8 shows that there are polynomials in $l_{1}$ which attain their norm at two points only, but do not approach it weakly.

The Banach space $l_{1}$, having the RNP, has many polynomials which attain their norm strongly, but in fact to attain the norm strongly is not too "strong" in $l_{1}$. From Proposition 4.5 and Corollary 3.10 we have the following result.

Proposition 4.6. If $P \in \mathcal{P}\left({ }^{k} l_{1}\right)$, then the following are equivalent:

(i) The norm is Fréchet differentiable at $P$.

(ii) The norm is Gateaux differentiable at $P$.

(iii) $P$ approaches its norm weakly.

Consider now the Banach space $l_{\infty}$. From Proposition 4.1, we know that there are no polynomials on $l_{\infty}$ attaining their norm strongly. Moreover, $l_{\infty}$ being a bidual, the following result holds.

Proposition 4.7. $\mathcal{P}\left({ }^{k} E^{* *}\right)=\mathcal{P}\left({ }^{k} E\right) \oplus \mathcal{P}_{0}\left({ }^{k} E^{* *}\right)$, where $\mathcal{P}_{0}\left({ }^{k} E^{* *}\right)$ is the subspace of all polynomials which vanish when restricted to $E$.

Proof. It is enough to consider $\pi: \mathcal{P}\left({ }^{k} E^{* *}\right) \rightarrow \mathcal{P}\left({ }^{k} E^{* *}\right)$ defined as $\pi(Q)=$ $L\left(Q_{\mid E}\right)$. Then $\pi$ is linear, continuous $(\|\pi\|=1)$, and $\pi^{2}=\pi$. Moreover Ker $\pi=\mathcal{P}_{0}\left({ }^{k} E^{* *}\right)$, and $\operatorname{Im} \pi \approx \mathcal{P}\left({ }^{k} E\right)$. 
Therefore every $Q \in \mathcal{P}\left(l_{\infty}\right)$ can be written as $R+T$, where $R=L\left(\left.Q\right|_{c_{0}}\right)$ and $\left.T\right|_{c_{0}}=0$. We have the following

COROLlary 4.8. $Q$ is $w^{*}$-sequentially continuous if and only if $T=0$.

Acknowledgments. This paper has been written when I was visiting the UCLA Mathematics Department. I wish to thank Professor T. Gamelin for his hospitality, as well as for the interesting talks we had. I would also like to thank Professors R. Aron, J. Gomez and J. G. Llavona for their comments about this subject. Finally I want to thank the anonymous referee for his suggestions about further developments of the results.

\section{References}

[1] M. D. Acosta, F. Aguirre and R. Payá, There is no bilinear Bishop-Phelps theorem, Israel J. Math. 93 (1996), 221-227.

[2] R. Alencar, R. M. Aron and S. Dineen, A reflexive space of holomorphic functions in infinitely many variables, Proc. Amer. Math. Soc. 90 (1984), 407-411.

[3] R. M. Aron, Compact polynomials and compact differentiable mappings between Banach spaces, in: Sém. P. Lelong 1974/75, Lecture Notes in Math. 524, Springer, 1976, 213-222.

[4] R. M. Aron and P. D. Berner, A Hahn-Banach extension theorem for analytic mappings, Bull. Soc. Math. France 106 (1978), 3-24.

[5] R. M. Aron, C. Finet and E. Werner, Some remarks on norm-attaining n-linear forms, in: Function Spaces (Edwardsville, 1994), Lecture Notes in Pure and Appl. Math. 172, Dekker, New York, 1995, 19-28.

[6] C. Benítez, Y. Sarantopoulos and A. Tonge, Lower bounds for norms of products of polynomials, Math. Proc. Cambridge Philos. Soc. 124 (1998), 395-408.

[7] Y. S. Choi and S. G. Kim, Polynomial properties of Banach spaces, J. Math. Anal. Appl. 190 (1995), 203-210.

[8] —, - Norm or numerical radius attaining multilinear mappings and polynomials, J. London Math. Soc. 54 (1996), 135-147.

[9] A. M. Davie and T. W. Gamelin, A theorem on polynomial-star approximation, Proc. Amer. Math. Soc. 106 (1989), 351-356.

[10] R. Deville, G. Godefroy and V. Zizler, Smoothness and Renormings in Banach Spaces, Pitman Monographs Surveys Pure Appl. Math. 64, Longman, 1993.

[11] J. Diestel, Sequences and Series in Banach Spaces, Springer, Berlin, 1984.

[12] S. Dineen, Complex Analysis on Infinite-Dimensional Spaces, Springer, London, 1999.

[13] -, A Dvoretzky theorem for polynomials, Proc. Amer. Math. Soc. 123 (1995), 28172821.

[14] J. Ferrera, J. Gómez and J. G. Llavona, On completion of spaces of weakly continuous functions, Bull. London Math. Soc. 15 (1983), 260-264.

[15] J. Gutiérrez, J. Jaramillo and J. G. Llavona, Polynomials and geometry of Banach spaces, Extracta Math. 10 (1995), 79-114.

[16] J. Jaramillo, A. Prieto and I. Zalduendo, The bidual of the space of polynomials on a Banach space, Math. Proc. Cambridge Philos. Soc. 122 (1997), 457-471. 
[17] J. Llavona, Approximation of Continuously Differentiable Functions, North-Holland Math. Stud. 130, North-Holland, 1986.

[18] J. Mujica, Complex Analysis in Banach Spaces, North-Holland Math. Stud. 120, North-Holland, 1986.

[19] R. A. Ryan, Dunford-Pettis properties, Bull. Acad. Polon. Sci. Sér. Sci. Math. 27 (1979), 373-379.

[20] C. Stegall, Optimization and differentiation in Banach spaces, Linear Algebra Appl. 84 (1986), 191-211.

Departamento de Análisis Matemático

Universidad Complutense de Madrid

28040 Madrid, Spain

E-mail: ferrera@mat.ucm.es

Received July 14, 1998

Revised version November 22, 2001 